\title{
Osteoid osteoma of the femoral head
}

\author{
H.K. Gupta ${ }^{1}$, K.D.Khare ${ }^{2}$
}

${ }^{1}$ Lecturer, ${ }^{2}$ Proffessor and Head, Department of Orthopaedics,College of Medical Sciences, Bharatpur, Chitwan, Nepal

\begin{abstract}
We present a rare case of intra-articular osteoid osteoma of the femoral head in the subarticular region in a 52 years old woman, treated with intralesional excision and bone grafting along with prophylactic internal fixation for impending stress fracture. At one year follow up, the patient has returned to her normal functional status. The patient was asymptomatic and without recurrence at one year follow up.
\end{abstract}

Keywords: Intra-articular, osteoid osteoma, intralesional excision.

\section{Introduction}

Osteoid osteoma is a common benign bone tumour accounting for approximately $12 \%$ of benign skeletal tumors. The pathological findings in osteoid osteoma was first described by Bergstrand in 1930 and Jaffe recognized it as a separate entity in his report of five cases in 1935 . $^{1}$

Most of the times , the diagnosis of osteoid osteoma is usually made as the clinical features and radiological features have been described well. The tumour is typically less than $2 \mathrm{~cm}$ in size occuring primarily in patients younger than $20 \mathrm{yrs}$. It presents with severe pain that worsens at night and relieved with aspirin or NSAID. Radiologically it has round and well demarcated nidus surrounded by reactive sclerosis of the surrounding bone which is smooth convex and homogenously dense. ${ }^{2}$ The frequent site is long bones of lower limbs usually in the metaphysis and diaphyses. Rarely it presents as an intra-articular lesion which presents as a diagnostic challenge.

Correspondence: H. K. Gupta

E-mail: hmntkg@yahoo.com

\section{Case Report}

A 52 year old women presented to our outdoor department with history of pain in the right hip for last 2 years. The pain had started spontaneously and was continuous dull aching type which was worse at night. There was no history of trauma, fever, pain in other joint. There was no significant past or family history. Gradually the pain increased in severity and she was taken for consultation to a general practitioner and was treated with ibuprofen and naproxen. The pain got relieved after taking medicine but recurred again after few hours. She continued taking same drugs on and off but the symptoms persisted.

Then she presented to our O.P.D with antalgic and trendelenberg gait, there was local tenderness in the hip, range of motion was restricted with end range pain in all planes. Straight leg raising was possible. There was no limb length discrepancy. Neurological examination found no abnormal results. Blood Counts and biochemical profile was within the reference range. 
Plain radiograph of pelvis showing both the hips AP view and right hip lateral view showed a radiolucent area in inferomedial area of the femoral head in subarticular area with dense central nidus and luscent areas surrounded by dense sclerotic bone (Fig-1). CT scan was also done confirming the similar findings (Fig-2), and the provisional diagnosis of osteoid osteoma was made.

The patient was then taken for surgical intervention. Under spinal anesthesia, the patient was put supine on the operating table with a sandbag below ipsilateral buttock. A $5 \mathrm{~cm}$ long incision made on the lateral aspect of the right thigh below trochantric flare. Under fluoroscopic guidance a guide wire was directed into the lesion and then drilled over it with $8 \mathrm{~mm}$ canulated reamer to reach the lesion. Then the lesion along with nidus was curreted out with a long curette and the tissues were sent for histopathological examination. Cortico-cancellous bone grafting was done, harvested from ipsilateral iliac crest. Then, internal fixation with two canulated cancellous screws was done for preventing stress fracture in the femoral neck region (Fig-3). Wound was closed under suction drainage in situ which was removed after $24 \mathrm{hrs}$ and sutures removed after 14 days. The patient was put on non weight bearing for initial 6 weeks and then allowed to bear weight on crutches and crutches removed after next 6 weeks. The patient was then followed up 3 monthly.

The histopathological report was consistent with the diagnosis of osteoid osteoma. The patient had complete pain relief of the tumour pain within one month of surgery. At one year follow up the patient was having painless hip with full range of movement and the lesion had healed radiologically.
Fig-1

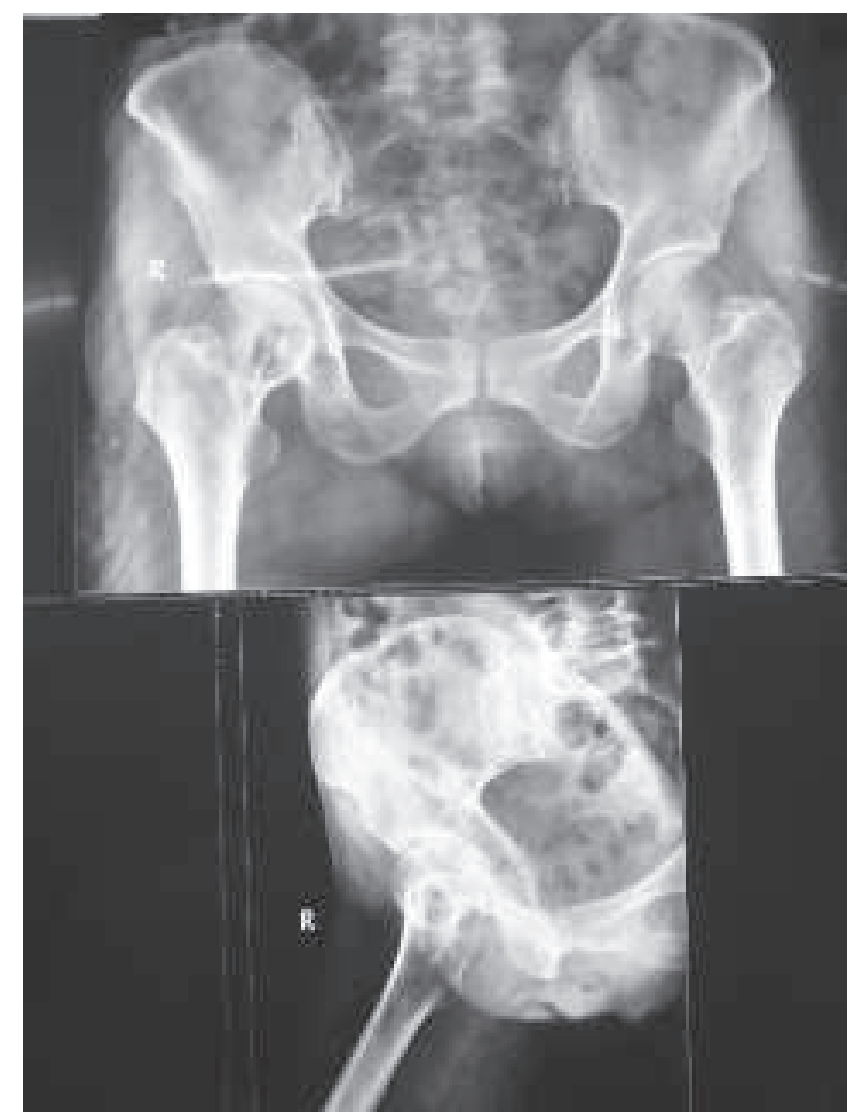

Fig-2

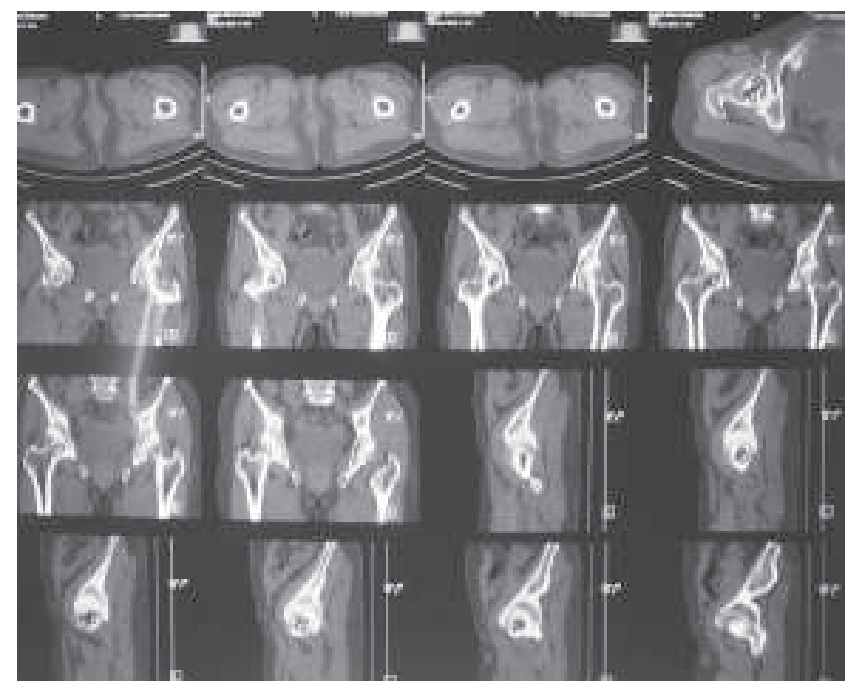




\section{Fig-3}

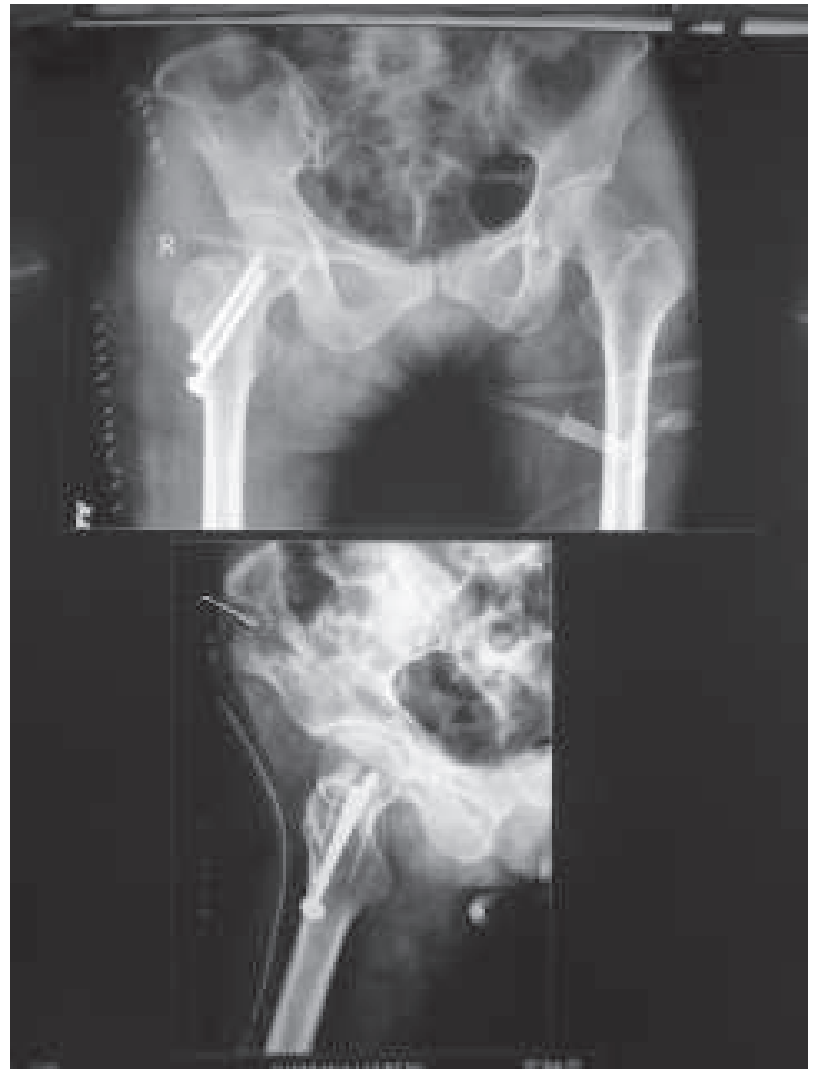

Fig-4

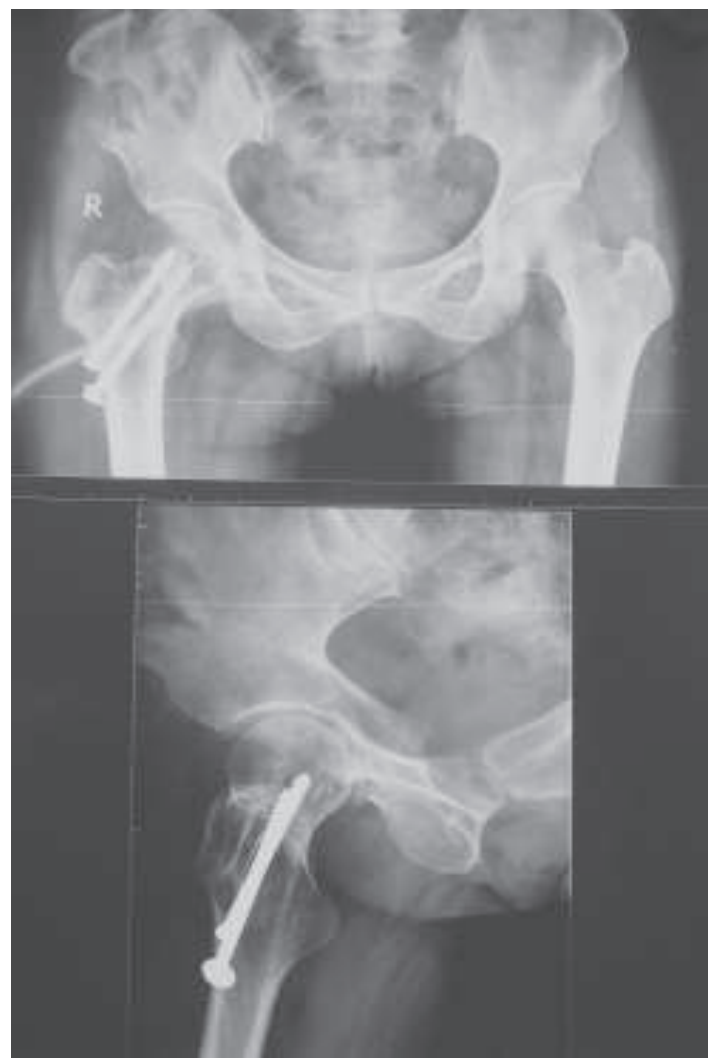

\section{Discussion}

Osteoid osteoma is benign bone forming tumour which involves both cortical and cancellous bones. The lesion of osteoid osteoma has predilection for femur and tibia and the leading symptom is pain. Osteoid osteoma of intra-articular type like in our case is rarely encountered, the diagnosis is often challenging because the radiological sign is less evident on plain radiographs. There is delay upto 2-2.5 years in diagnosis as per some reports. ${ }^{3}$ The investigation of choice is $\mathrm{CT}$ scan which is superior to MRI in identifying even small lesions and differentiate between normal trabecular bone and nidus. $^{4}$

There are some evidences that osteoid osteoma can regress spontaneously and also there is also a general consensus that the surgical en-bloc excision is the treatment of choice for osteoid osteoma and the incomplete excision would lead to recurrence. ${ }^{5}$ Recently minimally invasive techniques such as arthroscopic removal, cryoablation, thermoablation moreover radiofrequency ablation have emerged as an alternative to conventional surgical excision with the success rate of $70-100 \% .{ }^{6}$ All these facilities are not universally available and when most of the advance methods of treatment are not available surgical excision remains the standard treatment of choice. In this case the patient had undergone surgical intervention and achieved painless hip at one year follow up and the check x-ray showed healed lesion (Fig-4).

\section{References}

1. M.S. Sherman. Osteoid osteoma. Review of the literature and report of thirty cases. J. Bone and Joint Surg., 1947; 29:918-30. 
Journal of College of Medical Sciences-Nepal,2011,Vol-7,No-2

2. P. Picci, J.M. Mirra. Osteoid osteoma. In:MIrra JM,Picci P, Gold RH,editors.Bone tumours:clinical,radiological, and pathologic correlations. Philadelphia:Lea \&Febiger;1989:226-48.

3. V.N. Cassar-Pullicino, I.W. Mccall, S. Wan. Intra-articular osteoid osteoma. Clin Radiol 1992;45:153-60.

4. M. Szendroi, K. Kolio, I. Antal et al : Intra-articular osteoid osteoma,Clinical features,imaging results, and comparison with extra articular localization.J Rheumatol 2004;31:957-64.

5. M.W. Regan, J.P. Galey, R.D. Oakeshott. Recurrent osteoid osteoma.Case report with ten year asymptomatic interval.Clin Orthop Related Res 1990;25:321-4.

6. Z.G. Papathanassiou, P. Megas, T. Petsas et al. Osteoid osteoma: diagnosis and treatment.Orthopaedics 2008;31:1118. 